\title{
Performance analysis and preliminary design optimization of a Small Particle Heat Exchange Receiver for solar tower power plants
}

\author{
Pablo Fernández ${ }^{\mathrm{a}, \mathrm{b}, 1}$, Fletcher J. Miller ${ }^{\mathrm{a}, *}$ \\ ${ }^{a}$ Department of Mechanical Engineering, San Diego State University, San Diego, CA 92182-1323, United States \\ ${ }^{\mathrm{b}}$ Escuela de Ingenierías Industriales, Universidad de Valladolid, 47011 Valladolid, Spain
}

A multidisciplinary design optimization of the $5 \mathrm{MW}_{\text {th }}$, demonstration-scale Small Particle Heat

Exchange Receiver (SPHER) developed under the U.S. DOE SunShot Initiative is presented. SPHER is a revolutionary, high-temperature central receiver designed to drive a Brayton cycle or combined-cycle CSP plant that is expected to increase the overall efficiency of solar plants. The design space considered here consists of the geometry of the window, the lateral wall angle of the receiver, and the radiative properties of the walls. As a result of the optimization, the receiver efficiency is increased by $6 \%$ with respect to the baseline design.

Keywords: Central receiver; Concentrated solar power; CSP; Multidisciplinary design optimization; Numerical-stochastic modeling; Small Particle Heat Exchange Receiver

* Corresponding author: Department of Mechanical Engineering, San Diego State University, San Diego, CA 92182-1323, United States. Tel.: +1 619-594-5791; fax: +1 619-594-3599.

E-mail address: fmiller@mail.sdsu.edu (F. J. Miller)

\section{Introduction}

While current central receiver plants utilize molten salts as heat transfer fluid and Rankine steam cycles in the power block, there is a goal to develop higher efficiency plants based on Brayton cycle (gas turbine) or combined-cycle operation. The advantages of this technology are apparent. First, it would lead to higher thermodynamic cycle efficiency (due to the much higher temperatures) as shown by Fernández and Miller (2014a, 2014b); second, it would require much less cooling water (usually scarce in hot regions); third, it is a more convenient alternative for modular plants; and forth, the air is an inert, non-

\footnotetext{
${ }^{1}$ Currently Chief Engineer in the Solar Energy Division at Cidaut Foundation - R\&D Center in Energy and Transportation, 47151 Boecillo, Spain.
} 
problematic heat transfer fluid (unlike molten salts, which give problems of solidification and thermal degradation).

To accomplish the goal of solar-powered Brayton cycles, new solar receivers are needed. One such receiver, first proposed by Hunt (1979), is the Small Particle Heat Exchange Receiver (SPHER). This concept is based on employing small carbon particles ( 200 $\mathrm{nm}$ in diameter) in an air stream to selectively and volumetrically absorb concentrated solar irradiation and produce outlet temperatures in excess of $1300 \mathrm{~K}$, with the corresponding advantages previously mentioned. Moreover, it generates much less pressure drop, is probably less costly to construct, and can withstand higher incident flux levels than current (tubular) receivers. The latter feature, together with the intimate mixing between gas and particles, offers many possibilities for solar chemistry as well (Z' Graggen and Steinfeld, 2009).

A schematic of the preliminary design of the $5 \mathrm{MW}_{\text {th }}$ Small Particle Heat Exchange Receiver previously used (Fernández et al., 2013a) is shown in Fig. 1. The nomenclature employed throughout the paper to refer to the different walls of the receiver is included in the figure. The mixture of air and carbon nanoparticles enters the rear of the receiver (blue arrows), travels towards the front while absorbing concentrated solar irradiation (which penetrates through the ellipsoidal window displayed in gleaming light gray, as illustrated by the yellow arrows), and finally exits the receiver going backwards through the central outlet tube (red arrows). This outlet tube extends from the back wall to $0.5 \mathrm{~m}$ before the window. The particles oxidize before exiting the receiver (Leoni et al., 2014), leaving clear air for the gas turbine. No separation process of the carbon particles is therefore required between the receiver and the turbine.

The flow path in Fig. 1 was first considered by Crocker (2012a), after Ruther (2010) found that countercurrent flow seemed to lead to the most efficient receiver. Those earlier studies lacked certain physics, such as the effects of the window and a real solar irradiation coming from the heliostat field, and were limited to a right cylindrical geometry. All these effects are included in the present paper.

Our previous work has focused on developing a robust multi-physics model of the receiver and optimizing the iterative solution procedure for the governing integro-partial differential equations. While simple parametric studies of the operating conditions (mass flow rate and time of the day) have been performed and published previously (Crocker and Miller, 2012a; Fernández et al., 2013a), no effort had been made to optimize the design of the receiver. This is crucial in the highly competitive energy market. For example, increasing the overall efficiency of a $100 \mathrm{MW}_{\mathrm{e}} \mathrm{CSP}$ plant by one percent would translate 
into a profit increase of the order of several million USD. Hence, in order to maximize the efficiency, reduce capital and O\&M costs, increase the lifespan of the different components, and reduce the Levelized Cost of Energy (LCOE), the design of the SPHER needs to be optimized from a multidisciplinary approach.

\section{Numerical-stochastic model}

A three-dimensional fluid flow and radiative heat transfer model developed by Fernández (2013b) is employed to simulate the $5 \mathrm{MW}_{\text {th }}$ SPHER. A coupled CFD solver and in-house Monte Carlo ray tracing (MCRT) method is used to solve the governing equations, just like in the first model by Crocker (2012b). Further developments of the model now make it possible to exactly model the concentrated solar radiation that reaches the window from the heliostat field, and simulate any axisymmetric geometry for the solar receiver. Moreover, this new software can accommodate flat, ellipsoidal and spherical cap windows.

On account of their small size ( $200 \mathrm{~nm}$ in diameter), the carbon nanoparticles are in thermal equilibrium with their environment (Yuen et al., 1986) and move as part of the air flow (Fernández, 2013b). Therefore, the air-particle mixture is treated as a single phase for modeling purposes. Due to added complexity, particle oxidation is not included yet, but will be included in future publications. Physically, this corresponds with a nitrogen carrier gas (Klein et al., 2008), which is an alternative for the closed-loop operation of the receiver.

\subsection{CFD model}

The steady-state Reynolds-averaged Navier Stokes and energy equations (Eq. (1-3)), together with the two equations of the SST $\kappa-\omega$ turbulence model with default model constants (Eq. (4-5)) and the corresponding constitutive relations (not shown for simplicity), are solved numerically by the CFD package ANSYS Fluent (segregated pressure-based solver). Gravity forces are not included in the model and buoyancy forces are thereby neglected. Since a strong thermal stratification is expected inside the solar receiver, it constitutes a complicated problem itself that should be first addressed separately once the oxidation model is fully coupled. In the actual receiver, swirl will be introduced to avoid this problem.

Similarly, the pressure work, kinetic energy, viscous dissipation and chemical reaction (oxidation) terms are negligible compared to the divergence of the radiative heat flux and are not included in the energy equation, Eq. (3). In particular, the kinetic energy term is of the order of $\partial\left(\rho u_{j} u^{2} / 2\right) / \partial x_{j} \sim 10^{-1} W / m^{3}$ and the oxidation term is $q_{o x} \sim 10^{3} \mathrm{~W} / \mathrm{m}^{3}$, while the divergence of the radiative heat flux is 
$\partial q_{j}^{r} / \partial x_{j} \sim 10^{6} \mathrm{~W} / \mathrm{m}^{3}$. As for the viscous dissipation, the Brinkman number in the SPHER is $r \sim 10^{-7}$, while it is necessary that $\mathrm{Br}>1$ for the viscous heating to be of the same order of magnitude than the conductive heat transfer (which it is itself much smaller than the radiative heat transfer).

$\frac{\partial \rho u_{i}}{\partial x_{i}}=0$

$\frac{\partial}{\partial x_{j}}\left(\rho u_{i} u_{j}\right)+\frac{\partial p}{\partial x_{i}}-\frac{\partial \tau_{j i}}{\partial x_{j}}-\frac{\partial}{\partial x_{j}}\left(-\rho \overline{u_{\imath}^{\prime} u_{j}^{\prime}}\right)=0$

$\frac{\partial}{\partial x_{j}}\left(\rho u_{j}\left[h-\frac{p}{\rho}\right]\right)-\frac{\partial}{\partial x_{j}}\left(k_{e f f} \frac{\partial T}{\partial x_{j}}\right)+\frac{\partial q_{j}^{r}}{\partial x_{j}}=0$

$\frac{\partial}{\partial x_{j}}\left(\rho u_{j} k\right)=\tilde{P}_{k}-\beta^{*} \rho k \omega+\frac{\partial}{\partial x_{j}}\left[\left(\mu+\sigma_{k} \mu_{t}\right) \frac{\partial k}{\partial x_{j}}\right]$

$\frac{\partial}{\partial x_{j}}\left(\rho u_{j} \omega\right)=\frac{\alpha^{*}}{v_{t}} \tilde{P}_{k}-\beta \rho \omega^{2}+\frac{\partial}{\partial x_{j}}\left[\left(\mu+\sigma_{\omega} \mu_{t}\right) \frac{\partial \omega}{\partial x_{j}}\right]+2\left(1-F_{1}\right) \frac{\rho \sigma_{\omega 2}}{\omega} \frac{\partial k}{\partial x_{j}} \frac{\partial \omega}{\partial x_{j}}$

Since the maximum Mach number of the air-particle mixture is around 0.02 , the flow is treated as incompressible and the Incompressible Ideal Gas Law from ANSYS Fluent is applied. The air-particle mixture is assumed to behave as a thermally perfect gas, and the Navier-Poisson law with the Stokes' hypothesis is used for the strain rate-stress relation. All the thermophysical properties of the air and the walls are modeled as piecewise functions of the temperature owing to the high temperature differences inside the receiver. A structured hexahedral mesh is utilized in both inlet and outlet zones (where the flow moves essentially parallel to the axis of symmetry) to prevent numerical diffusion and improve the accuracy of the solution, while a non-structured tetrahedral mesh is used for the recirculation zones. The number of mesh cells for the different designs of the SPHER is shown in Table 1. A mesh independence study conducted by Crocker (2012b) showed that the computed receiver efficiency is independent of the number of grid cells, as long as a minimum (and relatively low) number of cells is used. This requirement is clearly met with the cells in Table 1.

Even neglecting the absorption and emission of the air-particle mixture, the flow in the outlet section is neither dynamically nor thermally fully developed $\left(L_{e, o t} \approx 20 \mathrm{~m} \gg 3 \mathrm{~m}=L_{o t}\right.$ for $\left.R e_{D, o t} \approx 2 \cdot 10^{5}\right)$. Therefore, a Dirichlet pressure-outlet boundary condition is used to allow axial diffusive transport on the outlet surface. As for the inlet section, a mass flow inlet boundary condition is employed to set the mass flow rate and calculate the pressure drop through the receiver. Finally, the window temperature is not calculated, but it is imposed as a Dirichlet boundary condition between $800 \mathrm{~K}$ and $850 \mathrm{~K}$, based on the 
preliminary results by Mecit et al. (2014). Full window coupling will be included in future publications.

\subsection{Radiative heat transfer model}

An in-house Monte Carlo ray tracing method (Fernández, 2013b; Fernández et al., 2014c; Ruther, 2010) is employed for radiative heat transfer due to the highly directional intensity distribution from the heliostat field (Mecit, 2013a; Mecit and Miller, 2013b) and the strong spectral dependence of the radiative properties of the particles (Ruther, 2010), which cannot be easily modeled by conventional numerical techniques such as the Spherical Harmonics or the Discrete Ordinates methods. Equation (6) gives the integro-differential form of the quasi-steady Radiative Transfer Equation (RTE) solved statistically by the Monte Carlo method, while Equations (7.a) and (7.b) show the boundary conditions on the walls and on the window, respectively. The right-hand side of Eq. (7.b), $\boldsymbol{i}_{\boldsymbol{w i n}, \lambda}(\widehat{\boldsymbol{s}})$, is the radiation intensity on the inner surface of the window, and is obtained from the heliostat field model developed by Mecit (2013a). This latter software is based on the Monte Carlo ray tracing method and is used to simulate the heliostat field of the National Solar Thermal Test Facility (Albuquerque, NM), where the SPHER will be tested. This way, the spatial, directional and wavelength dependence of the concentrated solar radiation at different times and days is exactly modeled by our software.

$\hat{\boldsymbol{s}} \cdot \nabla i_{\lambda}(\hat{\boldsymbol{s}})=\kappa_{\lambda} i_{b \lambda}-\left[\kappa_{\lambda}+\sigma_{s, \lambda}\right] i_{\lambda}(\hat{\boldsymbol{s}})+\frac{\sigma_{s, \lambda}}{4 \pi} \int_{4 \pi} i_{\lambda}\left(\hat{\boldsymbol{s}}^{\prime}\right) \Phi_{\lambda}\left(\hat{\boldsymbol{s}}, \hat{\boldsymbol{s}}^{\prime}\right) d \Omega^{\prime}$

$i_{\lambda}(\hat{\boldsymbol{s}})=\varepsilon_{\lambda}^{\prime} i_{b, \lambda}\left(T_{w}\right)+\int_{2 \pi} \rho_{\lambda}^{\prime \prime}\left(\hat{s}^{\prime}, \hat{s}\right) i_{\lambda}\left(\hat{\boldsymbol{s}}^{\prime}\right) \cos \theta^{\prime} d \Omega^{\prime} \quad \hat{\boldsymbol{s}} \cdot \widehat{\boldsymbol{n}}>0$ and $\forall \hat{\boldsymbol{s}}^{\prime} \cdot \widehat{\boldsymbol{n}}<0$
$i_{\lambda}(\widehat{\boldsymbol{s}})=i_{w i n, \lambda}(\widehat{\boldsymbol{s}})$
$\widehat{\boldsymbol{s}} \cdot \widehat{\boldsymbol{n}}>0$

Turbulence-radiation interactions have been neglected since the optically thin fluctuation approximation applies (Modest, 2011). The absorption and scattering properties of the carbon particles are calculated using Mie theory, since it constitutes the only satisfactory model for the interaction

between electromagnetic waves and spheres whose size parameter $\xi=\pi d / \lambda$ is of the order $10^{-2}-10^{2}$. Mie theory predicts a strong spectral dependence of the optical properties of the particles, which is crucial to reach high efficiencies by acting in a similar fashion to selective surfaces: Particles absorb radiation very well at solar wavelengths and emit poorly at longer (infrared) wavelengths (see Fig. 2). The gas phase is modeled as radiatively non-participating due to the negligible amount of $\mathrm{CO}_{2}$ generated in the receiver, which leads to $\alpha_{\mathrm{CO}_{2}} \approx 0$ vs. $\alpha_{\text {part }}=0.99$ for the solar spectrum and the axial path length. 
While the outlet tube is a three-dimensional volume in the CFD model (to allow axial, radial and circumferential heat conduction), it is treated as an infinitely thin, opaque surface in the radiation model. This hypothesis is acceptable due to the very small thickness of the outlet tube in comparison to the dimensions of the receiver.

\subsection{Coupling between the CFD model and the radiative heat transfer model}

The energy equation -solved numerically by ANSYS Fluent- depends on the divergence of the radiative heat flux (third term in Eq. (3)), while the Radiative Transfer Equation -solved stochastically by the Monte Carlo method- depends on the temperature field $\left(i_{b \lambda}=\sigma T^{4} / \pi, \kappa_{\lambda}, \sigma_{s, \lambda}, \varepsilon_{\lambda}^{\prime}\right.$ and $\rho_{\lambda}^{\prime \prime}$ are functions of temperature). In particular, the energy equation and the RTE are coupled through Eq. (8). In the SPHER model, Eq. (8) is implemented via User-Defined Functions (UDFs) and the CFD and MCRT solvers iterate alternately until convergence. The adaptive solution procedure was optimized to prevent numerical oscillations and reduce the CPU time by two orders of magnitude compared to the previous twodimensional version of the code developed by Crocker (2012b, 2014).

$\frac{\partial q_{j}^{r}}{\partial x_{j}}=\int_{0}^{\infty} \kappa_{\lambda}\left(4 \pi i_{b \lambda}-\int_{4 \pi} i_{\lambda} d \Omega\right) d \lambda$

\section{Design optimization methodology}

In PDE-constrained optimization problems, such as the design optimization of the $5 \mathrm{MW}_{\text {th }}$ SPHER, the objective function depends on the simulation results (the velocity and temperature fields), which in turn depend on the design variables through the governing equations (the Navier-Stokes equations and the RTE). It is therefore computationally very expensive to compute the derivatives of the objective function with respect to the design variables. Possible approaches to that end, such as adjoint methods ${ }^{2}$ or finite differences, are much beyond the scope of this project or would require an unaffordable CPU time. This implies that gradient-based methods cannot be employed for the design optimization of the $5 \mathrm{MW}_{\text {th }} \mathrm{Small}$ Particle Heat Exchange Receiver. Therefore, the design space will be explored in a finite number of points (rather than finding the actual solution), which leads to a NP-hard discrete optimization problem. Thus, the design space should be cleverly defined to avoid introducing irrelevant or insensitive variables. An informal formulation of the problem is presented below.

\footnotetext{
${ }^{2}$ The adjoint operator of the RTE is such that $L^{*}(\boldsymbol{x}, \Omega)=L(\boldsymbol{x},-\Omega)$. Hence, our in-house Monte Carlo ray tracing software could, with small modifications, be employed to solve the adjoint thermal radiation problem.
} 
Design space: The design space considered here consists of the lateral wall angle of the receiver, the geometry of the window, and the radiative properties of the walls. Other variables, such as window diameter, receiver depth, etc. are not varied since we are constrained by the $5 \mathrm{MW}_{\mathrm{th}}$ input of the heliostat field for the experimental test, and those other variables are functions of that.

Constraints: The constraints are based on material limits (for example, the maximum operating temperature of aluminium oxide is around $1533 \mathrm{~K}$ ), ensuring the mechanical integrity of the quartz window, and other technical issues. Some of these constraints are imposed via a penalty method as they depend on the simulation results.

Objective function: A wide variety of objective functions can be defined, such as the receiver efficiency at a particular time, the thermal energy collected by the receiver throughout the day, or even throughout the year. For a more multidisciplinary approach, the generation cost of the electricity could be minimized, for which the cost of the different components and their expected life span need to be known. In this paper, the receiver efficiency at 12:00 PM on the Spring equinox will be employed as the objective function since the cost of the components cannot be accurately estimated yet. Objective functions that consider the weighted-average efficiency at different times and days would be more convenient to elucidate the best design, but would require a prohibitive CPU time.

Optimization technique: The design space is explored via parametric studies. This way, it will be possible to identify important and sensitive variables, determine approximate design variable ranges to meet material limits, and obtain a first estimate of the optimum design and of the maximum efficiency that the $5 \mathrm{MW}_{\text {th }}$ SPHER can achieve.

The design parameters and the operating conditions of the baseline design are based on preliminary results by Fernández (2013b) and are collected in Table 2. The tilt angle denotes the angle between the symmetry axis of the receiver and the horizontal plane (positive if pointing upwards, negative if pointing downwards). The thermal resistance reported corresponds with the combined thermal resistance of the conduction inside the walls (including the insulation) and the exterior convection to the atmosphere. In other words, the interior convection to the air-particle mixture is not included. Hence, the equivalent convective heat transfer coefficient from the inner surface of the receiver walls to the atmosphere is as expressed in Eq. (9). 
$h^{*}=\left(\int_{0}^{t_{w}} \frac{d x}{k_{w}(x)}+\frac{1}{h_{\text {ext }}}\right)^{-1}=\frac{1}{\mathfrak{R}}$

The solar input is $4.25 \mathrm{MW}_{\mathrm{th}}$, instead of $5 \mathrm{MW}_{\mathrm{th}}$, since the simulations were performed for the Spring equinox instead of for the Summer solstice. A higher input power could be obtained just by increasing the size of the heliostat field. The inlet temperature $(700 \mathrm{~K})$ corresponds with the expected inlet temperature when using the receiver in a recuperated, single-shaft gas turbine engine (Fernández and Miller, 2014b; Kitzmiller and Miller, 2011).

\section{Results}

\subsection{Wall properties optimization}

The infinite degrees of freedom necessary to describe the spectral dependence of the radiative properties of the walls can be reduced essentially to four types of properties, denoted by P1, P2, P3 and P4:

- P1: High absorptivity in the solar spectrum and low emissivity at longer, infrared wavelengths (e.g. selective coatings of solar collectors used in parabolic trough plants or domestic hot water systems.)

- P2: High absorptivity and emissivity in the whole spectrum (e.g. a blackbody).

- P3: Low absorptivity in the solar spectrum and high emissivity at infrared wavelengths (e.g. aluminum oxide used for insulation.)

- P4: Low absorptivity and emissivity in the entire spectrum.

An approximation of the spectral absorptivity of these four types of radiative properties is shown in Fig. 3. The actual spectral absorptivity of aluminum oxide, used in the simulations, is also represented in the figure (Ruther, 2010). The directional behavior is assumed diffuse in all cases (though not physically realistic for P4.) Finally, since the walls are at local thermodynamic equilibrium, the spectral emissivity equals the spectral absorptivity.

Table 3 shows a summary of the simulation results with the radiative properties P2, P3, and P4. The results for the properties $\mathrm{P} 1$ are not shown as they lead to unacceptable wall temperatures above even those of the blackbody case. From Table 3 it can be concluded that the radiative properties P3 (aluminum oxide) show the best compromise between wall temperature and thermal efficiency. Moreover, aluminum oxide walls would provide thermal insulation on the inside of the receiver. 
It should be noted that the thermal efficiency is defined as the useful thermal output divided by the solar power that goes through the window and enters the receiver (Eq. (10)). Hence, the losses due to absorption and reflection in the window are not included in the definition of thermal efficiency, but rather they are accounted for by the so-called optical efficiency of the receiver (the transmissivity of the window.) It is important to note also that the low thermal efficiency and the high temperature of the lateral wall are mainly due to the reduced diameter and simple, right-cylindrical geometry of the baseline design. Optimized geometries will dramatically reduce the wall temperature and increase the thermal efficiency, as will be discussed later. Finally, and as expected, the pressure drop is essentially independent of the radiative properties of the walls.

$\eta_{\text {thermal }}=\frac{\dot{Q}_{\text {output }}}{\dot{Q}_{\text {input }}}$

\subsection{Window geometry optimization}

As demonstrated by Saung and Miller (2014), a curved window is required to withstand the mechanical loading caused by the pressurized environment inside the receiver $\left(5\right.$ bar in the $5 \mathrm{MW}_{\text {th }}$ SPHER considered here, and about 5-10 bar when eventually introducing the receiver in the high-pressure side of a gas turbine engine). The material selected for the window is fused quartz, particularly HOQ-310, due to its very high compressive strength (around $1100 \mathrm{MPa}$ ) and selective optical behavior: High transmissivity in the solar spectrum and low transmissivity at infrared wavelengths. A fused quartz window will perform well as long as only small tensile stresses are allowed to develop (thereby the curved geometry.) Moreover, its low coefficient of thermal expansion accounts for its remarkable ability to undergo large, rapid temperature changes, such as during cloudy transient periods ${ }^{3}$, without cracking. The spectral refractive index and absorptive index of HOQ-310 (Mecit, 2013a) were obtained from the transmissivity data provided by the vendor.

Regarding its shape, spherical-cap and ellipsoidal windows are considered (Fig. 4), and this comparison is the matter of this section. The latter is simply a prolate spheroid with ratio between principal axes $\boldsymbol{r}=\sqrt{\mathbf{2}}$. As for mechanical considerations, the ellipsoidal geometry would eliminate tensile stresses and the window would be entirely in compression (Mande, 2011). Moreover, it may be

\footnotetext{
${ }^{3}$ In reality, the heliostat field would be gradually covered by a transient cloud, and the temperature gradient with respect to time would be softened.
} 
preferable from a sealing perspective. Spherical windows, however, are much easier to fabricate and polish than ellipsoidal shapes as they are a portion of a sphere. Previous studies conducted by Mecit (2013a) showed that the optical efficiency of spherical-cap windows has one global minimum at $45^{\circ}$ cap angle and then increases in both directions (towards $0^{\circ}$ and towards $90^{\circ}$ ). The optical efficiency of the ellipsoidal window equals the one of a $70^{\circ}$ cap angle window. These results refer to the transmittance of concentrated solar irradiation from the heliostat field to the inside of the receiver. However, the transmission of radiation from the inside to the outside of the receiver constitutes the main loss mechanism (Fernández et al, 2013a) and needs to be considered as well. Thus, the optimum window geometry will be a compromise between both effects. Furthermore, the efficiency is only one of the many aspects to be considered in this multidisciplinary decision-making process of the window shape.

A summary of the simulation results with the ellipsoidal window $(\boldsymbol{r}=\sqrt{\mathbf{2}})$ and the $45^{\circ}$ spherical-cap window is shown in Table 4. As expected (Mecit, 2013a), the optical efficiency is greater with the ellipsoidal window, but the overall receiver efficiency (optical + thermal) is higher with the $45^{\circ}$ sphericalcap geometry. This result is mainly because the ellipsoidal window penetrates deeper into the solar receiver than the $45^{\circ}$ spherical-cap window and the radiative losses are thereby higher. The maximum wall and outlet tube temperature is essentially independent of the window geometry. With full window coupling, some small differences might be noticed. Again, the high wall temperature and low thermal efficiency are due to the reduced diameter and the right-cylindrical geometry of the baseline design. The pressure drop is kept constant in both cases since the position of the outlet tube was chosen to maintain a distance to the window of $0.5 \mathrm{~m}$. Finally, the total radiation absorbed by the ellipsoidal window is $5 \%$ greater than with the $45^{\circ}$ spherical-cap window, but the absorption per unit area is smaller with the ellipsoidal window due to its higher surface area. Also, the vast majority of the radiation absorbed from the receiver side is emitted from the inside of the SPHER, and only a negligible amount of back-scattered and back-reflected radiation from the heliostat field is absorbed.

The optimum window geometry should be a compromise between efficiency, mechanical behavior, manufacturing issues, and economic aspects. Hence, the $45^{\circ}$ spherical-cap window is likely preferred over the ellipsoidal window as it provides higher efficiency and is less expensive and easier to manufacture. Further stress (Saung and Miller, 2014) and thermal (Mecit et al., 2014) analyses of the two geometries are currently under study.

\subsection{Receiver geometry optimization}


To simplify the analysis, only the angle between the front wall and the initial part of the lateral wall or, equivalently, between the back surface and the end of the lateral wall- is varied (see Fig. 5 for greater clarity.) The length and front diameter of the receiver are kept constant ( $3 \mathrm{~m}$ and $2 \mathrm{~m}$, respectively) in all the designs. This way, the continuous function necessary to describe the generatrix of the solar receiver (infinite degrees of freedom) is reduced to only one degree of freedom. In particular, the two geometries illustrated in Fig. 5 were simulated, which correspond with the cases of $0^{\circ}$ and $45^{\circ}$ lateral wall angle. The choice of these designs is based on preliminary studies of the distribution of solar irradiation on the walls for different receiver geometries (Fernández, 2013b).

The main simulation outputs for the two geometries analyzed are collected in Table 5, while Fig. 5 shows the temperature fields inside the receiver. The $45^{\circ}$ lateral wall-angle design maximizes the thermal efficiency and minimizes the temperature of the walls, which is now acceptable unlike in previous sections (the maximum operating temperature of aluminium oxide is about $1533 \mathrm{~K}$ ). This result is expected since the optical thickness provided by this geometry in different directions approximates the directional distribution of the incoming radiation. The outlet temperature of the air is greater than the maximum temperature of the walls, and therefore material limits are no a constraint to the maximum temperature that can be reached. The $45^{\circ}$ lateral wall-angle receiver also considerably reduces the radiation absorbed by the window, mainly due to the lower temperature level (Fig. 5) and the smaller view factor from the walls to the window. This is important to keep the window cool and, in turn, ensure the integrity of the quartz, reduce the thermal losses and allow the use of an anti-reflective coating. The pressure drop is limited to $173 \mathrm{~Pa}$, which is well below the value of tubular receivers and does not diminish the thermodynamic efficiency of the gas turbine (note the receiver is located between the compressor and the turbine). The outlet tube temperature is not a concern as it can be easily reduced just by distancing it from the window.

Therefore, the sloped wall geometry is preferred for the $5 \mathrm{MW}_{\text {th }}$ SPHER to be constructed and tested at the National Solar Thermal Test Facility (Albuquerque, USA). For the eventual implementation of the SPHER in commercial CSP plants, an exhaustive cost analysis should be conducted to elucidate the best receiver geometry from a technico-economic point of view. Nevertheless, since the SPHER efficiency directly affects the plant efficiency -while its cost is only a small fraction of the total capital cost of the plant-, the $45^{\circ}$ lateral wall-angle design is probably also preferred for commercial applications. Note finally that the $45^{\circ}$ geometry has greater cross sectional area than the cylindrical design, which would 
increase the residence time of the particles and could lead to premature oxidation, thereby limiting the outlet temperature. That effect will be explored in future work once the oxidation model is fully coupled.

\section{Discussion and future work}

The $45^{\circ}$ lateral wall-angle receiver, the $45^{\circ}$ spherical-cap window, and the aluminium oxide walls are preferred over the other alternatives considered in this paper. While interactions between design variables are not properly captured by parametric studies, it is thought that they are small in our design space and the conclusions inferred for each variable are of general validity.

It is of great interest to analyze the origin of the thermal losses in order to further optimize the receiver. Table 6 collects the contribution of the three main loss mechanisms in all the designs presented in this paper. All other loss mechanisms are negligible compared to these three. Several conclusions can be drawn from these numbers:

- The emission from the mixture of air and carbon particles is the main loss mechanism in all the designs analyzed except in the case of black walls (wall properties P2). In that case, the emission from the inner part of the lateral walls prevails over other mechanisms because of their high absorptivity and emissivity $\left(\alpha_{\lambda}=\varepsilon_{\lambda}=1\right)$.

- Back-reflected (by the walls) and back-scattered (by the air-particle mixture) solar irradiation is significantly reduced when using black walls as they act as perfect absorbers and no back-reflection $\operatorname{occurs}\left(\alpha_{\lambda}=1\right)$.

- These three types of thermal losses are considerably reduced with the $45^{\circ}$ lateral wall-angle geometry (with respect to the baseline design) because of the lower air-particle mixture temperature and the smaller view factor from the lateral wall to the window. Further optimization of the lateral wall is recommended for the next phase of the project.

- The three types of losses increase with the ellipsoidal window, probably due to the fact that this window shape penetrates deeper into the receiver and the view factors from the air-particle mixture and from the walls to the window are greater. While this result has only been demonstrated for the $0^{\circ}$ lateral wall-angle case, it is probably true for any wall angle.

- The losses due to emission from the air-particle mixture are lower in the preliminary design used in previous publications (Fernández et al., 2013a) than in any of the designs analyzed in this paper. After 
careful investigation of this fact, it is attributable to the greater receiver diameter employed previously. It is thus suggested to use a slightly larger receiver diameter in future designs. Note also that the low back-scattered and back-reflected solar radiation in the preliminary design is due to the black walls employed in that design.

The extremely high temperature in some fluid regions of the receiver is caused by the lack of particle oxidation in the current model. These temperatures are not physically realistic since particles would immediately oxidize and no radiation could be absorbed in those zones. This, in turn, would increase the thermal efficiency of the receiver since that part of the incoming radiation would be instead absorbed by a lower-temperature zone, and a more homogeneous temperature field would be obtained. (The more uniformly distributed radiation is absorbed, the lower the radiative losses are since the emissive power is a convexe function of the temperature.) In addition, the receiver geometry has not been optimized from a fluid flow point of view. This increases the volume of the hot spots due to poor advection in those zones. While flow optimization was not part of this work, it will be addressed when the oxidation model is fully coupled, since it will be then possible to introduce swirl and buoyancy forces in the model.

The outlet temperature of the SPHER will probably be limited by the temperature at which the particles fully oxidize, although the efficiency could be maintained by increasing the mass flow rate even if premature oxidation occurred. Note, however, that this strategy would reduce the solar share since the natural gas consumption would be increased due to both higher carbon particle requirements (to maintain the mass loading and optical thickness with an increased mass flow rate) and higher fuel demand in the combustor (to achieve the turbine inlet temperature desired). Particle oxidation is currently being added to the three-dimensional SPHER model to quantify this effect, and the results will be included in future publications.

It is suggested to locate the air-particle inlets in different axial positions of the lateral walls for two main reasons. First, the operational flexibility (important in pilot plants) would increase since different strategies and flow patterns could be analyzed. This in turn would improve the robustness of the design with respect to the oxidation kinetics. And second, these lateral inlets would avoid no-particle zones near the walls ${ }^{4}$, a feature necessary to keep the wall temperature below its upper temperature limit. A disadvantage of this multiple-inlet design is that the small holes of the inlets would increase the pressure

\footnotetext{
${ }^{4}$ If no action was taken in the $45^{\circ}$ lateral wall-angle design, a no-particle zone would appear near the lateral walls due to particle oxidation and the lack of convective transport in that zone to replace the oxidized particles.
} 
drop through the receiver. Hence, they should be designed to ensure that the pressure drop remains low and the thermodynamic efficiency of the gas turbine is not significantly reduced. Other possible future work to improve the Small Particle Heat Exchange Receiver includes use of a high-temperature antireflective coating on the window, a further design optimization (more points and new degrees of freedom), a flow optimization considering buoyancy effects, and the analysis of the cocurrent flow direction (a preliminary study about this topic was conducted by Fernández (2013b)).

As previously mentioned, the window temperature is not calculated in the current model. Instead, it is imposed as a boundary condition of $850 \mathrm{~K}$ if $r \leq 0.3 m$ or $800 \mathrm{~K}$ if $r>0.3 m$ ( $r$ denotes the radial coordinate of the receiver). For this window temperature, the radiative source term in the window turns out to be positive, i.e. the actual temperature is greater than $800-850 \mathrm{~K}$. This implies that more radiation will actually be emitted by the window, more useful power will be collected by the receiver, and the actual thermal efficiency will be higher than the one predicted here. Therefore, the values of the thermal efficiency presented in this paper are only to be understood as relative or comparative values between different designs, but not as absolute values. For example, the actual thermal efficiency of the $45^{\circ}$ lateral wall-angle design is probably close to $90 \%$, instead of the predicted $85 \%$.

\section{Conclusions}

A multidisciplinary design optimization of a $5 \mathrm{MW}_{\text {th }}$ Small Particle Heat Exchange Receiver for central receiver solar power plants was presented. This new solar receiver, currently being developed under the U.S. DOE SunShot Program, aims to heat air to temperatures in excess of $1300 \mathrm{~K}$ and use this high-temperature energy to drive a Brayton cycle or a combined Brayton/Rankine cycle. The design space considered consisted of the lateral wall angle of the receiver, the geometry of the window, and the radiative properties of the walls. The aluminum oxide walls, which would also serve as thermal insulation, showed the best compromise between wall temperature and thermal efficiency compared to the other three main types of radiative properties that could be employed. As for the window geometry, the SPHER efficiency is higher with a $45^{\circ}$ spherical-cap window than with an ellipsoidal window, while the wall temperature is virtually independent of its shape. In addition, the $45^{\circ}$ spherical-cap window is less expensive and easier to manufacture. Finally, regarding the receiver geometry, the $45^{\circ}$ lateral wall- 
angle design was the best geometry analyzed as it maximizes the thermal efficiency and minimizes the wall temperature.

As a result of this design optimization, the receiver efficiency was increased by $6 \%$ with respect to the baseline design. In particular, a thermal efficiency of $85 \%$, an optical efficiency of $92 \%$, an outlet temperature of $1486 \mathrm{~K}$, and a maximum wall temperature of $1325 \mathrm{~K}$ were predicted. The actual thermal efficiency, however, is expected to be higher $(\sim 90 \%)$ when the window temperature is calculated, instead of being imposed as a boundary condition as in the current model. As a comparison, tubular receivers are predicted to have much lower efficiencies and much greater pressure drops when operating at these temperatures (Kolb, 2011).

\section{Acknowledgments}

The authors gratefully acknowledge the U.S. Department of Energy for providing funding for this research through the SunShot Initiative (Award \#DE-EE0005800). Moreover, we would like to thank Aerojet Rocketdyne, Solar Turbines and Thermaphase Energy for their collaboration in the project. We are also grateful to all the members of the Combustion and Solar Energy Laboratory at San Diego State University, especially to Ryan Contois and Ahmet Murat Mecit for their assistance in the preparation of this paper.

\section{Nomenclature}

$d$

Brinkman number (-)

$F_{1}$ carbon particle diameter $(\mu \mathrm{m})$

blending function

$h \quad$ specific enthalpy $\left(\mathrm{J} \mathrm{kg}^{-1}\right)$

$h^{*} \quad$ convective heat transfer coefficient equivalent to the combined thermal resistance of the receiver walls and the exterior convection $\left(\mathrm{W} \mathrm{m}^{-2} \mathrm{~K}^{-1}\right)$

$h_{\text {ext }} \quad$ convective heat transfer coefficient from the outer surface of the receiver to the atmosphere (W $\left.\mathrm{m}^{-2} \mathrm{~K}^{-1}\right)$

$i_{\lambda} \quad$ spectral intensity $\left(\mathrm{W} \mathrm{m}^{-2} \mu \mathrm{m}^{-1} \mathrm{sr}^{-1}\right)$

$i_{b \lambda} \quad$ blackbody spectral intensity $\left(\mathrm{W} \mathrm{m}^{-2} \mu \mathrm{m}^{-1} \mathrm{sr}^{-1}\right)$

$i_{\text {win }, \lambda} \quad$ spectral intensity on the inner surface of the window coming from the heliostat field (W $\mathrm{m}^{-2}$ $\left.\mu \mathrm{m}^{-1} \mathrm{sr}^{-1}\right)$ 


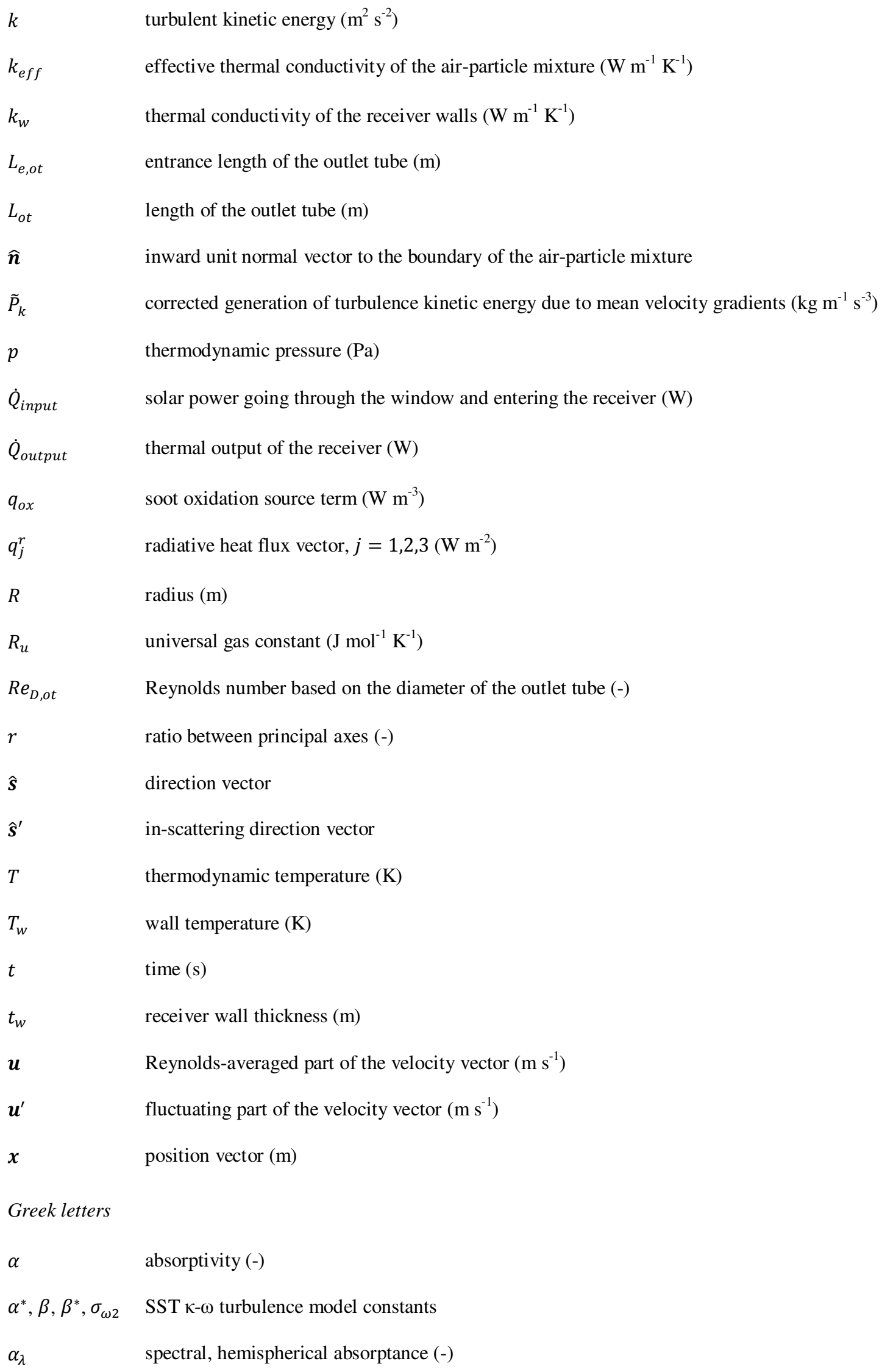




\begin{tabular}{|c|c|}
\hline$\varepsilon_{\lambda}$ & spectral, hemispherical emittance (-) \\
\hline$\varepsilon_{\lambda}^{\prime}$ & spectral, directional emittance (-) \\
\hline$\eta_{\text {thermal }}$ & thermal efficiency of the receiver (-) \\
\hline$\theta$ & zenith angle (rad) \\
\hline$\kappa_{\lambda}$ & spectral absorption coefficient $\left(\mathrm{m}^{-1}\right)$ \\
\hline$\lambda$ & wavelength $(\mu \mathrm{m})$ \\
\hline$\mu$ & molecular dynamic viscosity $\left(\mathrm{kg} \mathrm{s}^{-1} \mathrm{~m}^{-1}\right)$ \\
\hline$\mu_{t}$ & turbulent eddy viscosity $\left(\mathrm{kg} \mathrm{s}^{-1} \mathrm{~m}^{-1}\right)$ \\
\hline$\xi$ & particle size parameter (-) \\
\hline$\rho$ & density of the air-particle mixture $\left(\mathrm{kg} \mathrm{m}^{-3}\right)$ \\
\hline$\rho_{\lambda}^{\prime \prime}$ & spectral, bidirectional reflection function $\left(\mathrm{sr}^{-1}\right)$ \\
\hline$\sigma$ & Stefan-Boltzmann constant $\left(\mathrm{W} \mathrm{m}^{-2} \mathrm{~K}^{-4}\right)$ \\
\hline$\sigma_{s, \lambda}$ & spectral scattering coefficient $\left(\mathrm{m}^{-1}\right)$ \\
\hline$\sigma_{k}$ & turbulent Prandtl number for $k(-)$ \\
\hline$\sigma_{\omega}$ & turbulent Prandtl number for $\omega(-)$ \\
\hline$\tau_{j i}$ & Reynolds-averaged viscous stress tensor $(\mathrm{Pa})$ \\
\hline$\Phi_{\lambda}$ & spectral scattering phase function $\left(\mathrm{sr}^{-1}\right)$ \\
\hline$\Omega$ & solid angle (sr) \\
\hline$\omega$ & specific dissipation rate $\left(\mathrm{s}^{-1}\right)$ \\
\hline
\end{tabular}

Manuscripts letters

$\mathfrak{R} \quad$ combined thermal resistance of the receiver walls and the exterior convection to the atmosphere $\left(\mathrm{K} \mathrm{m}^{2} \mathrm{~W}^{-1}\right)$

Subscripts

$\mathrm{a} / \mathrm{p} \quad$ air-particle mixture

Abbreviations

CFD computational fluid dynamics

CSP concentrated solar power

DOE Department of Energy

LCOE Levelized Cost of Energy 
MCRT Monte Carlo ray tracing

RTE radiative transfer equation

SPHER Small Particle Heat Exchange Receiver

\section{References}

Crocker, A., Miller, F.J., 2012a. Coupled Fluid Flow and Radiation Modeling of a Cylindrical Small Particle Solar Receiver. Proceedings of the ASME $20126^{\text {th }}$ International Conference on Energy Sustainability, ESFuelCell2012-91235, San Diego, CA. $\underline{\text { http://proceedings.asmedigitalcollection.asme.org/proceeding.aspx?articleid=1719575 }}$

Crocker, A., 2012b. Coupled Fluid Flow and Radiation Modeling of a Small Particle Solar Receiver. Master's Thesis, Department of Mechanical Engineering, San Diego State University, San Diego, CA. http://sdsu-dspace.calstate.edu/handle/10211.10/5902

Crocker, A., Miller, F.J., Fernández, P., 2014. Coupled Fluid Flow and Radiation Modeling of a Cylindrical Small Particle Solar Receiver. Journal of Heat Transfer (in preparation for submission).

Fernández, P., Miller, F.J., Crocker, A., 2013a. Three-Dimensional Fluid Dynamics and Radiative Heat Transfer Modeling of a Small Particle Solar Receiver. Proceedings of the ASME $20137^{\text {th }}$ International Conference on Energy Sustainability, ES2013-18149, Minneapolis, MN. http://proceedings.asmedigitalcollection.asme.org/proceeding.aspx?articleid=1797409

Fernández, P., 2013b. Numerical-Stochastic Modeling, Simulation and Design Optimization of Small Particle Solar Receivers for Concentrated Solar Power Plants. Proyecto Fin de Carrera, University of Valladolid, Spain. http://solar.sdsu.edu/Master's\%20Projects/PabloFernandezdelCampo/PabloThesis.pdf

Fernández, P., Miller, F.J., 2014a. Assessment of the Overall Efficiency of Gas Turbine-Driven CSP Plants Using Small Particle Solar Receivers. Energy Procedia 49, 334-343. Presented at the $19^{\text {th }}$ SolarPACES Conference, Las Vegas, NV, USA. http://www.sciencedirect.com/science/article/pii/S1876610214004901

Fernández, P., Miller, F.J., 2014b. Analysis of a Solar-Fossil Hybrid Gas Turbine Plant Based on a Small Particle Heat Exchange Receiver. J. Sol. Energy Eng. (submitted).

Fernández, P., Miller, F.J., Crocker, A., 2014c. Application and optimization of the Monte Carlo method for radiation heat transfer in axisymmetric geometries. International Journal of Heat and Mass 
Transfer (in preparation for submission).

Hunt, A.J., 1979. A New Solar Thermal Receiver Utilizing Small Particles. Proceedings of the International Solar Energy Society Conference, Atlanta, GA.

Kitzmiller, K., Miller, F. J., 2011. Thermodynamic Cycles for Small Particle Solar Receivers used in Concentrating Solar Power Plants. J. Sol. Energy Eng. 133(3), 031014. $\underline{\text { http://proceedings.asmedigitalcollection.asme.org/proceeding.aspx? } \text { articleid=1607611 }}$

Klein, H.H., Rubin, R., Karni, J., 2008. Experimental Evaluation of Particle Consumption in a Particle Seeded Solar Receiver. J. Sol. Energy Eng. 130(1), 011012. http://solarenergyengineering.asmedigitalcollection.asme.org/article.aspx?articleid=1473913

Kolb, G.J., 2011. An Evaluation of Possible Next-Generation High-Temperature Molten-Salt Power Towers. SAND2011-9320, Sandia National Laboratories, Albuquerque, NM. http://prod.sandia.gov/techlib/access-control.cgi/2011/119320.pdf

Leoni, M., Frederickson, L., Miller, F., 2014. Oxidation Rate Analysis of Carbon Nanoparticles for a Small Particle Heat Exchange Receiver. Proceedings of the ASME $20148^{\text {th }}$ International Conference on Energy Sustainability, ESFuelCell2014-6648, Boston, MA.

Mande, O.K., 2011. Window and Seal Design for a Small Particle Solar Receiver. Master's Thesis, Department of Mechanical Engineering, San Diego State University, San Diego, CA. http://sdsu$\underline{\text { dspace.calstate.edu/handle/10211.10/1703 }}$

Mecit, A.M., 2013a. Optical Analysis and Modeling of a Window for Small Particle Solar Receiver Using the Monte Carlo Ray Trace Method. Master's Thesis, Department of Mechanical Engineering, San Diego State University, San Diego, CA. http://sdsu-dspace.calstate.edu/handle/10211.10/4259

Mecit, A.M., Miller, F.J., 2013b. Optical Analysis of a Window for Solar Receivers Using the Monte Carlo Ray Trace Method. Proceedings of the ASME $20137^{\text {th }}$ International Conference on Energy Sustainability, ES2013-18186, Minneapolis, MN. http://proceedings.asmedigitalcollection.asme.org/proceeding.aspx?articleid=1797410

Mecit, A. M., Miller, F.J., Whitmore, A., 2014. Optical Analysis and Thermal Modeling of a Window for a Small Particle Solar Receiver. Energy Procedia 49, 457-467. Presented at the $19^{\text {th }}$ SolarPACES Conference, Las Vegas, NV, USA.

http://www.sciencedirect.com/science/article/pii/S1876610214005037

Modest, M.F., 2011. Radiative Heat Transfer, second ed., Academic Press, San Diego. 
Ruther, S.J., 2010. Radiation Heat Transfer Simulation of a Small Particle Solar Receiver Using the Monte Carlo Method. Master's Thesis, Department of Mechanical Engineering, San Diego State University, San Diego, CA. http://sdsu-dspace.calstate.edu/handle/10211.10/578

Saung, E., Miller, F.J., 2014. Dome Window and Mount Design for a $5 \mathrm{MW}_{\text {th }}$ Solar Receiver. Energy

Procedia 49, 514-523. Presented at the $19^{\text {th }}$ SolarPACES Conference, Las Vegas, NV, USA.

http://www.sciencedirect.com/science/article/pii/S1876610214005098

Yuen, W.W., Miller, F.J., Hunt, A.J., 1986. Heat Transfer Characteristics of a Gas-Particle Mixture under

Direct Radiant Heating. International Communications in Heat and Mass Transfer 13(2), 145-154.

$\underline{\text { http://www.sciencedirect.com/science/article/pii/0735193386900540 }}$

Z'Graggen, A., Steinfeld, A., 2009. Heat and mass transfer analysis of a suspension of reacting particles

subjected to concentrated solar irradiation - Application to the steam-gasification of carbonaceous

materials. International Journal of Heat and Mass Transfer 52(1-2), 385-395.

http://www.sciencedirect.com/science/article/pii/S0017931008003335

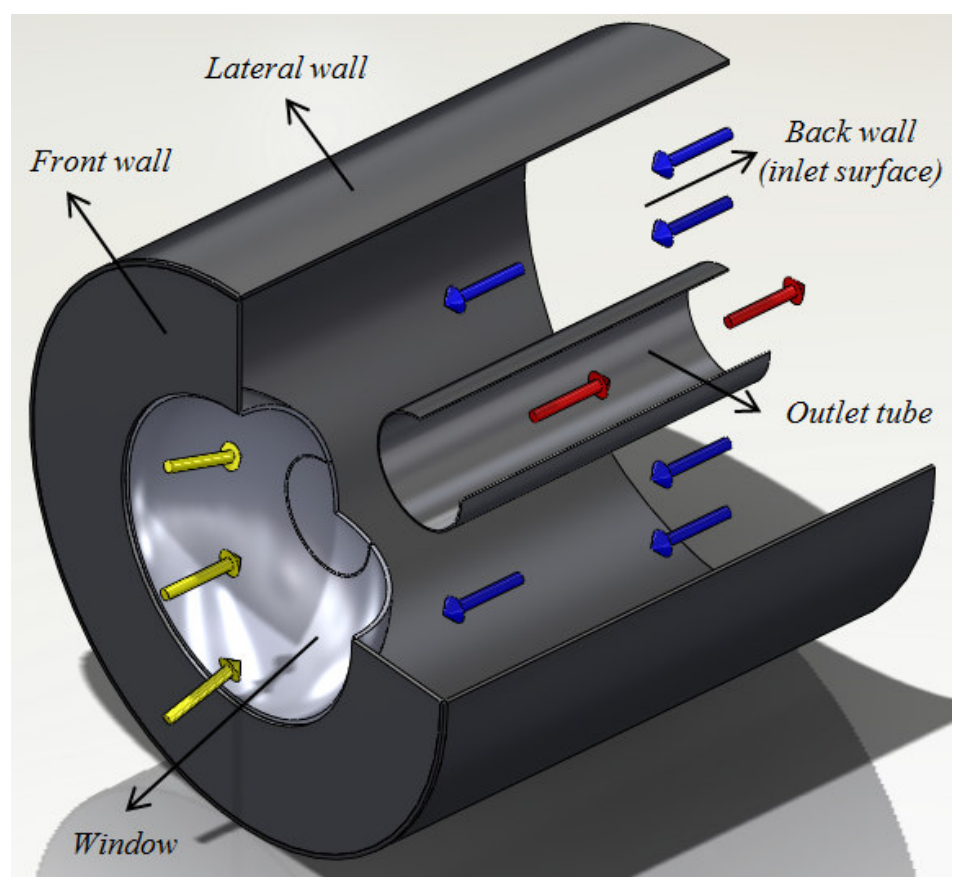

Fig. 1. Schematic of the preliminary design of the $5 \mathrm{MW}_{\text {th }}$ Small Particle Heat Exchange Receiver used by Fernández et al. (2013a). Yellow arrows: solar irradiation; blue arrows: air-particle mixture inlet; red arrows: air-particle mixture outlet. The back wall is not displayed for clarity. 


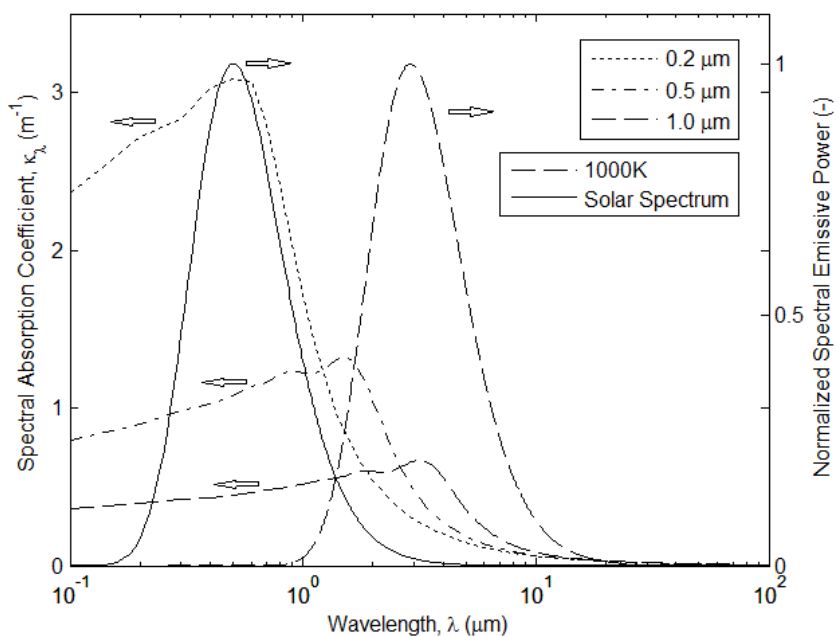

Fig. 2. Spectral absorption coefficient of the air-particle mixture $\left(0.5 \mathrm{~g} / \mathrm{m}^{3}\right)$ for different particle diameters. The normalized blackbody spectral emissive power at $1000 \mathrm{~K}$ (emission from inside of the receiver) and $5777 \mathrm{~K}$ (approximate solar spectrum) are also shown. Only the relative $x$-axis position is wished to be pointed out.

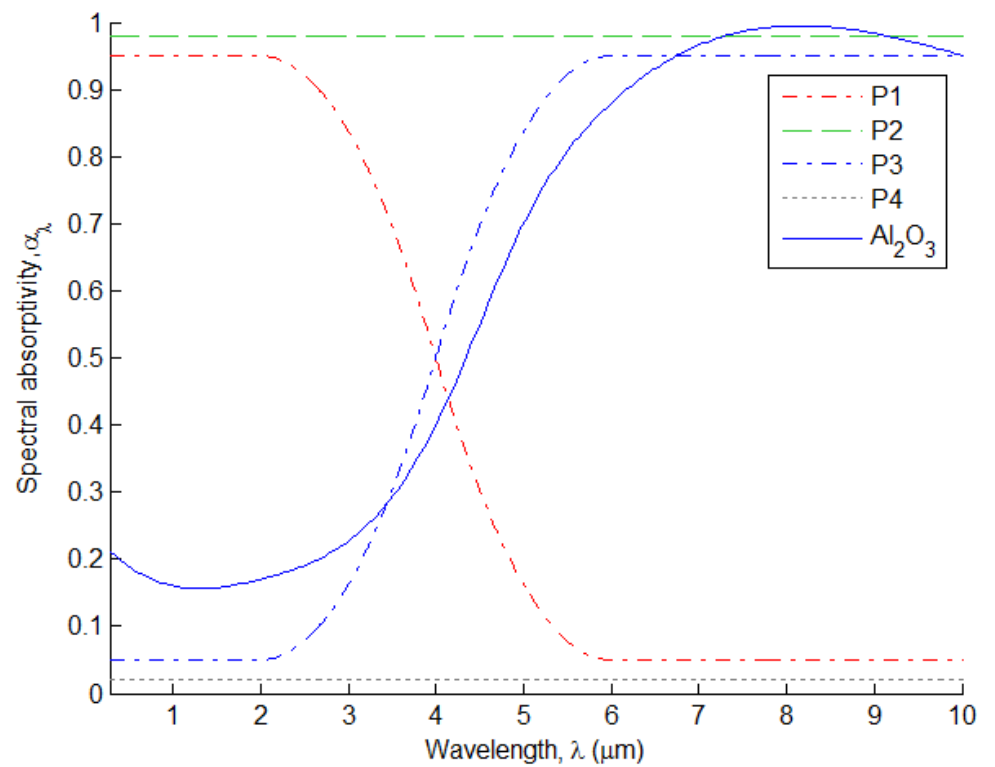

Fig. 3. Schematic representation of the spectral absorptivity of the four types of radiative properties of the walls considered. The actual properties of aluminum oxide $\left(\mathrm{Al}_{2} \mathrm{O}_{3}\right)$ are displayed as well. The spectral emissivity equals the spectral absorptivity. 


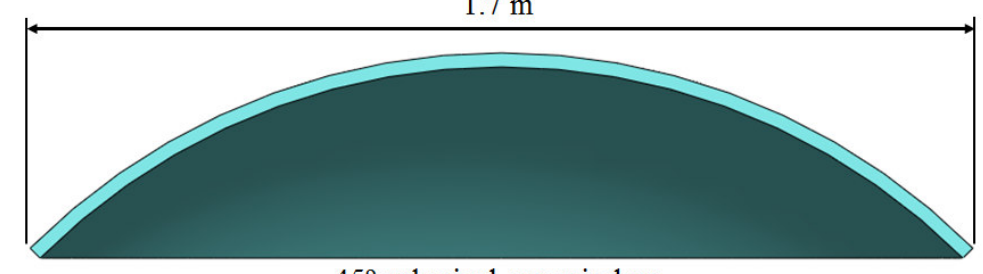

$45^{\circ}$ spherical-cap window

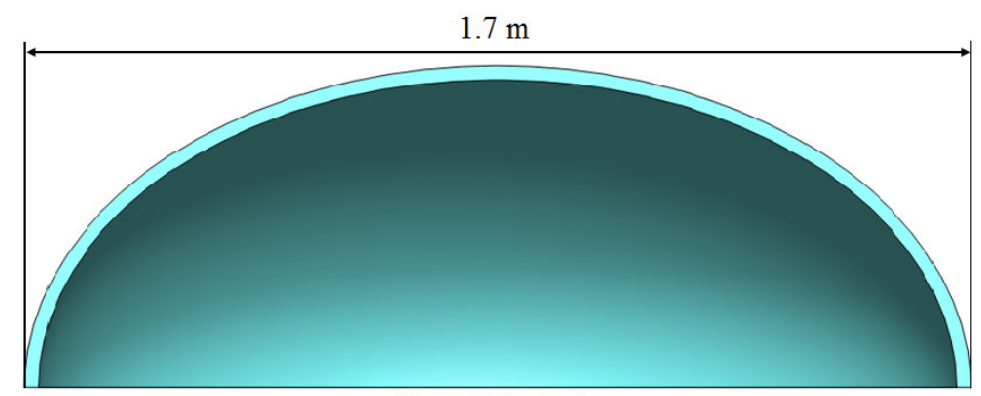

Ellipsoidal window

Fig. 4. $45^{\circ}$ spherical-cap window (top) and ellipsoidal window (bottom).

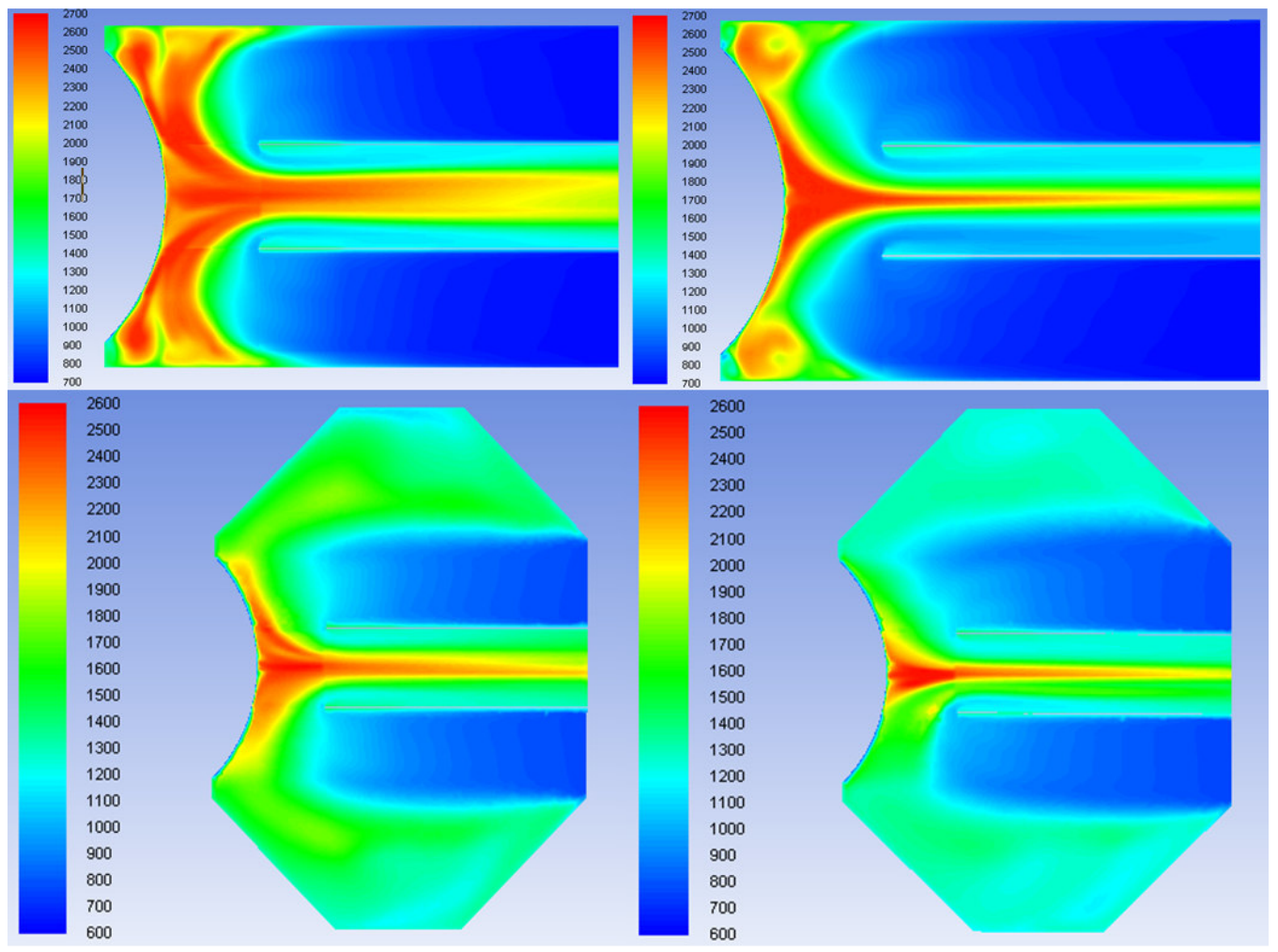

Fig. 5. Temperature field $(\mathrm{K})$ in horizontal section on the left and vertical section on the right. The first row corresponds with the $0^{\circ}$ lateral wall angle and the second row with the $45^{\circ}$ lateral wall angle. The color scale varies between both designs. Note also that the second design is at a smaller scale than the first one for an easier visualization of the temperature field (both designs are the same length: $3 \mathrm{~m}$ ) 


\begin{tabular}{cc}
\hline Design & Cells \\
\hline Baseline & $1,656,610$ \\
Ellipsoidal Window & $1,216,320$ \\
$45^{\circ}$ Lateral Wall Angle & $2,346,852$ \\
\hline
\end{tabular}

Table 1

Number of cells of the CFD model of the different SPHER designs analyzed in this paper.

\begin{tabular}{|c|c|c|c|}
\hline Design Parameter & Value & Design Parameter & Value \\
\hline $\begin{array}{l}\text { Receiver: } \\
\text { - Shape } \\
\text { - Length } \\
\text { - Front diameter } \\
\text { - Tilt angle } \\
\text { Window: } \\
\text { - Material } \\
\text { - Shape } \\
\text { - Diameter } \\
\text { - Thickness } \\
\text { - Temperature }\end{array}$ & $\begin{array}{c}\text { HOQ- } 310 \\
\text { Spherical cap ( } 45^{\circ} \text { cap angle) } \\
1.7 \mathrm{~m} \\
2.54 \mathrm{~cm} \\
\text { Between } 800 \mathrm{~K} \text { and } 850 \mathrm{~K} \\
\text { (depending on radial position) }\end{array}$ & $\begin{array}{l}\text { Walls: } \\
\text { - Material } \\
\text { - Thermal resistance } \\
\text { Mass flow rate } \\
\text { Inlet temperature } \\
\text { Solar irradiation: } \\
\text { - Time } \\
\text { - Day } \\
\text { - Solar input } \\
\text { Operating pressure } \\
\text { Inlet particle mass loading } \\
\text { Particle diameter } \\
\text { Ambient temperature }\end{array}$ & $\begin{array}{c}\text { Aluminum oxide (Fig. 3) } \\
2 \mathrm{~m}^{2}-\mathrm{K} / \mathrm{W} \\
4 \mathrm{~kg} / \mathrm{s} \\
700 \mathrm{~K} \\
12.00 \mathrm{PM} \\
\text { Spring equinox } \\
4.25 \mathrm{MW}_{\text {th }} \\
5 \mathrm{bar} \\
0.5 \mathrm{~g} / \mathrm{m}^{3} \\
200 \mathrm{~nm} \\
293 \mathrm{~K}\end{array}$ \\
\hline
\end{tabular}

Table 2

Design parameters and operating conditions of the baseline design considered in this paper.

\begin{tabular}{lccc}
\hline & $\mathrm{P} 2$ & $\mathrm{P} 3$ & $\mathrm{P} 4$ \\
\hline Radiative properties employed to simulate this case & $\alpha_{\lambda}=1$ & $\mathrm{Al}_{2} \mathrm{O}_{3}$ (Fig. 3) & $\alpha_{\lambda}=0.1$ \\
Thermal efficiency of the receiver & $77.5 \%$ & $79.6 \%$ & $80.5 \%$ \\
Outlet temperature & $1414 \mathrm{~K}$ & $1433 \mathrm{~K}$ & $1440 \mathrm{~K}$ \\
Maximum temperature: & & & \\
$-\quad$ Walls & $\sim 1725 \mathrm{~K}$ & $\sim 1600 \mathrm{~K}$ & $\sim 1750 \mathrm{~K}$ \\
$-\quad$ Outlet tube & $\sim 1600 \mathrm{~K}$ & $\sim 1450 \mathrm{~K}$ & $\sim 1500 \mathrm{~K}$ \\
Pressure drop & $164 \mathrm{~Pa}$ & $165 \mathrm{~Pa}$ & $165 \mathrm{~Pa}$ \\
\hline
\end{tabular}

Table 3

Summary of simulation results with the different types of wall radiative properties considered.

\begin{tabular}{lcc}
\hline & $45^{\circ}$ Spherical-Cap Window & Ellipsoidal Window \\
\hline Efficiency of the receiver: & & \\
$-\quad$ Optical (transmissivity of the window) & $92.1 \%$ & $93.1 \%$ \\
$-\quad$ Thermal (useful power / solar power that & $79.6 \%$ & $76.0 \%$ \\
$\quad$ enters the receiver) & $73.4 \%$ & $70.8 \%$ \\
$-\quad$ Overall (optical + thermal) & $1433 \mathrm{~K}$ & $1407 \mathrm{~K}$ \\
Outlet temperature & & \\
Maximum temperature: & $\sim 1600 \mathrm{~K}$ & $\sim 1600 \mathrm{~K}$ \\
$-\quad$ Walls & $1450 \mathrm{~K}$ & $\sim 1450 \mathrm{~K}$ \\
$-\quad$ Outlet tube & $274 \mathrm{~kW}$ & $288 \mathrm{~kW}$ \\
Radiation absorbed by the window: & $33 \mathrm{~kW}$ & $33 \mathrm{~kW}$ \\
$-\quad$ From the heliostat field side & $241 \mathrm{~kW}$ & $255 \mathrm{~kW}$ \\
$-\quad$ From the solar receiver side & $165 \mathrm{~Pa}$ & $166 \mathrm{~Pa}$ \\
Pressure drop & & \\
\hline
\end{tabular}

Table 4

Summary of simulation results with the ellipsoidal and the $45^{\circ}$ spherical-cap window.

\begin{tabular}{lcc}
\hline & $0^{\circ}$ Lateral Wall Angle & $45^{\circ}$ Lateral Wall Angle \\
\hline Thermal efficiency & $79.6 \%$ & $85.5 \%$
\end{tabular}




$\begin{array}{lcc}\text { Outlet temperature } & 1433 \mathrm{~K} & 1486 \mathrm{~K} \\ \text { Maximum temperature: } & \sim 1600 \mathrm{~K} & \sim 1325 \mathrm{~K} \\ -\quad \text { Walls } & \sim 1450 \mathrm{~K} & \sim 1500 \mathrm{~K} \\ -\quad \text { Outlet tube } & 274 \mathrm{~kW} & 215 \mathrm{~kW} \\ \text { Radiation absorbed by the window: } & 33 \mathrm{~kW} & 33 \mathrm{~kW} \\ -\quad \text { From the heliostat field side } & 241 \mathrm{~kW} & 182 \mathrm{~kW} \\ -\quad \text { From the solar receiver side } & 165 \mathrm{~Pa} & 173 \mathrm{~Pa}\end{array}$

Table 5

Table 5
Summary of simulation results with the two geometries of solar receiver considered here.

\begin{tabular}{|c|c|c|c|c|c|c|}
\hline Loss Mechanism & $\begin{array}{c}\text { Previous design } \\
\text { (Fig. 1) }\end{array}$ & Baseline & Wall Properties P2 & Wall Properties P4 & Ellipsoidal Window & $\begin{array}{l}45^{\circ} \text { Lateral } \\
\text { Wall Angle }\end{array}$ \\
\hline Emission from the air-particle mixture & $123 \mathrm{~kW}$ & $339 \mathrm{~kW}$ & $191 \mathrm{~kW}$ & $363 \mathrm{~kW}$ & $372 \mathrm{~kW}$ & $224 \mathrm{~kW}$ \\
\hline $\begin{array}{l}\text { Back-scattered (air-particle mixture) and } \\
\text { back-reflected (walls) solar radiation }\end{array}$ & $73 \mathrm{~kW}$ & $168 \mathrm{~kW}$ & $77 \mathrm{~kW}$ & $184 \mathrm{~kW}$ & $223 \mathrm{~kW}$ & $95 \mathrm{~kW}$ \\
\hline $\begin{array}{l}\text { Emission from the inner part of the } \\
\text { lateral walls }\end{array}$ & $72 \mathrm{~kW}$ & $57 \mathrm{~kW}$ & $275 \mathrm{~kW}$ & $40 \mathrm{~kW}$ & $73 \mathrm{~kW}$ & $27 \mathrm{~kW}$ \\
\hline Total & $268 \mathrm{~kW}$ & $564 \mathrm{~kW}$ & $544 \mathrm{~kW}$ & $587 \mathrm{~kW}$ & $668 \mathrm{~kW}$ & $347 \mathrm{~kW}$ \\
\hline
\end{tabular}

Contribution of the three main loss mechanisms in all the designs presented in this paper and in the previous design used by Fernández et al. (2013a). 
Fig. 1. Schematic of the preliminary design of the $5 \mathrm{MW}_{\text {th }}$ Small Particle Heat Exchange Receiver used by Fernández et al. (2013b). Yellow arrows: solar irradiation; blue arrows: air-particle mixture inlet; red arrows: air-particle mixture outlet.

Fig. 2. Schematic representation of the spectral absorptivity of the four types of surface radiative properties considered. The actual properties of aluminum oxide $\left(\mathrm{Al}_{2} \mathrm{O}_{3}\right)$ are displayed as well. The spectral emissivity equals the spectral absorptivity in diffuse surfaces at local thermodynamic equilibrium.

Fig. 3. $45^{\circ}$ spherical-cap window (top) and ellipsoidal window (bottom).

Fig. 4. Temperature field $(\mathrm{K})$ in horizontal section on the left and vertical section on the right. The first row corresponds with the $0^{\circ}$ wall angle and the second row with the $45^{\circ}$ wall angle. The color scale varies between both designs. Note also that the second design is at a smaller scale than the first one for an easier visualization of the temperature field (both designs are the same length: $3 \mathrm{~m}$ ). 